International Journal of Applied Mathematics

Volume 29 No. 2 2016, 219-226

ISSN: 1311-1728 (printed version); ISSN: 1314-8060 (on-line version)

doi: http://dx.doi.org/10.12732/ijam.v29i2.6

\title{
ON PRICING EUROPEAN CALL OPTION ON \\ EXPONENTIAL LÉVY MODEL WITH JUMPS IN INTEREST RATE
}

\author{
Michael C. Anyanwu ${ }^{1}$, Georgina O. Kalu ${ }^{2}$ \\ ${ }^{1,2}$ Department of Mathematics \\ Michael Okpara University of Agriculture \\ Umudike, Abia State, NIGERIA
}

\begin{abstract}
This article presents a simple but efficient method for pricing European call option in exponential Lévy model when the interest rate is stochastic with jumps. We relax two assumptions in the Black and Scholes model: geometric Brownian motion for asset price and constant interest. The asset price is assumed to be given by a more general stochastic process, the Lévy process, and the interest rate in the market has stochastic paths with jumps. The resulting partial-integro differential equation(PIDE) for the option price is reduced to a system of first order partial differential equation, which is easier to solve. Hence, the option price and its sensitivities are easily obtained.
\end{abstract}

AMS Subject Classification: 60G51, 60J75, 91G20

Key Words: option, call option, stochastic interest rate, exponential Lévy process

\section{Introduction}

In the Black and Scholes [3] model, the price of European option is obtained in closed-form, under the assumption that the only source of uncertainty is the price of the underlying asset, which is assumed to follow a geometric Brownian motion with constant drift and constant volatility. It is also assumed in this model that the risk-free interest rate in the market remains constant throughout the journey of the option. These assumptions have been criticized widely by

Received: January 11, 2016

(C) 2016 Academic Publications

${ }^{\S}$ Correspondence author 
many, in that they contribute to the inability of the model to capture the important features of asset returns. In this regard, there have being some efforts to relax some of these assumptions.

The assumption of single source of uncertainty in the Black and Scholes model was relaxed by proposing stochastic volatility models e.g. Heston [8], Stein and Stein [14]. In these models, the volatility of the asset price is allowed to follow another stochastic differential equation with a different standard Brownian motion. On the other hand, to relax the assumption of geometric Brownian motion, models which have jumps in their paths were proposed. In these models, the asset price is assumed to be given by an exponential Lévy process. Exponential Lévy processes are of two categories: the first category is the jump-diffusion models which are a combination of a Brownian motion and a compound Poisson process with given distribution of jump sizes, e.g jump-diffusion process of Merton [13], and double exponential jump process of Kou [9]. The second category has only the jump component, e.g the variance gamma process of Madan and Milne [11], and Normal Inverse Gaussian process of Barndorff-Nielsen [2], etc.

In using these Lévy processes for financial modeling, either the interest rate is constant, or it varies stochastically until the option matures e.g. see ([5], [1], [6], [10]) and bibliographies therein. In models with constant interest rate, it is possible to obtain a closed-form formula for the option price, while in models with stochastic interest rate, close-form formula is impossible, and numerical methods are used.

To price a European option in exponential Lévy model with stochastic interest rate, we assume that the asset price $X_{t}$ is a Lévy process of exponential type, and the interest rate in the market is not only stochastic but exhibits jumps in its paths.Further, we assume that the interest rate $r_{t}$ is a stochastic function of a Markov process $Y_{t}: r=r\left(Y_{t}\right)$, with state space $D=\mathbb{R}$, where $Y_{t}$ is given by the mean-reverting process of CIR-type [7]. We convert the resulting PIDE for the option price to a system of first order partial differential equation. This system is easier to solve to obtain the option price and its sensitivities.

The rest of this paper is organized as follows: For the rest of Section 1, we present some of the basic facts from the theory of Lévy processes that are needed in the formulation of the model. In Section 2, we formulate the model, and formula for the option price and its sensitivities. In Section 3, we implement the model and conclude. 


\subsection{Brief on Lévy Processes}

Lévy processes are stochastic processes with stationary and independent increments. A general 1-dimensional Lévy process can be represented as

$$
X_{t}=\gamma t+\omega_{t}+N_{t}+\lim _{\epsilon \rightarrow 0} M_{t}^{\epsilon},
$$

where $\omega_{t}$ is a 1-dimensional Brownian motion, $\gamma \in \mathbb{R}, N_{t}$ is a compound Poisson process which includes the jumps of $X_{t}$, and $M_{t}^{\epsilon}$ is a compensated compound Poisson process which includes the jumps of $X_{t}$ with $\epsilon<\left|\Delta X_{t}\right| \leq 1$. The characteristic function of the distribution of $X_{t}$ can be represented in the form

$$
E\left[e^{\imath \eta X_{t}}\right]=e^{-t \psi(\eta)}, \quad \eta \in \mathbb{R}, t \geq 0 .
$$

The function $\psi$ is called the characteristic exponent for $X$. The general form of characteristic exponent of any Lévy process can be deduced from the LévyKhintchine formula

$$
\psi(\eta)=\frac{\sigma^{2}}{2} \eta^{2}-i b \eta+\int_{\mathbb{R} \backslash 0}\left(1+i \eta x 1_{[-1,1]}(x)-e^{i x \eta}\right) F(d x),
$$

where $\sigma \geq 0, b \in \mathbb{R}$, and $F$ is a measure on $\mathbb{R} \backslash 0$ satisfying

$$
\int_{\mathbb{R} \backslash 0}\left\{|x|^{2}, 1\right\} F(d x)<\infty .
$$

As an example, we use a variance gamma (VG) process, which is a pure jump process with density of jumps given by

$$
F(d x)=c_{+} e^{\lambda_{-} x} x^{-1} 1_{(0,+\infty)}(x) d x+c_{-} e^{\lambda_{+} x} x^{-1} 1_{(-\infty, 0)}(x) d x .
$$

Here, $1_{(a, b)}$ is an indicator function of the interval $(a, b)$. The coefficients $c_{+}$ (respectively, $c_{-}$) gives the intensity of upwards jumps(respectively, downward jumps). The characteristic exponent of the VG process can be obtained from (2):

$$
\psi(\eta)=c\left[\ln \left(-\lambda_{-}-i \eta\right)-\ln \left(-\lambda_{-}\right)+\ln \left(\lambda_{+}-i \eta\right)-\ln \left(\lambda_{+}\right)\right] .
$$

\section{Pricing European Option with Stochastic Interest Rate}

Let $\left(\Omega, \mathbb{B}, P, f_{t}\right)$ be the underlying filtered probability space, where $f_{t}$ is the filtration which represents the information available up to time $t$. The interest rate in the market is a stochastic function of a Markov process $Y_{t}: r_{t}=r\left(Y_{t}\right)$ with state space $D=\mathbb{R}$. The underlying asset price is modeled as $S_{t}=\exp \left(X_{t}+\right.$ 
$Y_{t}$ ), where $X_{t}$ is an exponential Lévy process. The processes $X_{t}$ and $Y_{t}$ are assumed to be strong solution to the stochastic differential equations

$$
\begin{gathered}
d X_{t}=\mu\left(Y_{t}\right) d t+d J_{t}^{x}, \\
\left.d Y_{t}=\alpha\left(\beta-Y_{t}\right) d t+\sigma \sqrt{(} Y_{t}\right) d \omega_{t}+d J_{t}^{y},
\end{gathered}
$$

where $d \omega_{t}$ is the increment of standard Brownian motion, $d J_{t}^{x}$ and $d J_{t}^{y}$ are increments of pure jump Lévy processes of exponential type $\left(\lambda_{-}, \lambda_{+}\right)$. We assume that the drift $\mu\left(Y_{t}\right)$ of $d X_{t}$ is affine in $Y_{t}$ such that $\mu\left(Y_{t}\right)=\mu_{0}+\mu_{1} Y_{t}$, where $\mu_{0}$ and $\mu_{1}$ are positive real constants, $\alpha>0$ is the coefficient of mean reversion, $\beta \geq 0$ is the long-run mean level of $Y_{t}$, and $\sigma>0$ is the volatility of $Y_{t}$.

Consider a European call option with strike $K$ and expiry date $T$, under the chosen risk-neutral measure $Q$ for pricing, the value $V(t, x, y)$, of this contingent claim is given by

$$
V(t, x, y)=E^{Q, x, y}\left[e^{-\int_{t}^{T} r\left(y_{s}\right) d s} G\left(X_{T}, Y_{T}\right)\right],
$$

where $G\left(X_{T}, Y_{T}\right)=e^{X_{T}+Y_{T}}-K$, is the payoff function. Applying the FeynmanKac theorem, we get that $V(t, x, y)$ satisfy the backward Kolmogorov equation:

$$
\left(\partial_{t}+\alpha(\beta-y) \partial_{y}+\frac{\sigma^{2} y}{2} \partial_{y}^{2}+\left(\mu_{0}+\mu_{1} y\right) \partial_{x}+L_{x}+L_{y}-y\right) V(t, x, y)=0,
$$

where $L_{x}$ and $L_{y}$ are the infinitesimal generators that act with respect to $x$ and $y$ respectively as

$$
L g(x)=\int_{\mathbb{R} \backslash 0}(g(x+y)-g(x)) F(d x) .
$$

Given that the coefficients in (6) are affine, we propose a solution of the form

$$
V(t, \eta, x, y)=\exp (A(\tau, \eta) x+B(\tau, \eta) y+C(\tau, \eta)),
$$

subject to $V(T, \eta, x, y)=e^{i \eta(x+y)}$, where $\tau=T-t$ is the time to maturity of the option. Substituting (7) into (6) and simplifying, we obtain a system of first order differential equations

$$
\begin{array}{r}
-A_{\tau}(\tau, \eta)=0, \\
-B_{\tau}(\tau, \eta)+\frac{\sigma^{2} B^{2}}{2}-\alpha B+\mu_{1} A-1=0, \\
-C_{\tau}(\tau, \eta)+\mu_{0} A+\alpha \beta B-\psi(-i A)-\psi(-i B)=0,
\end{array}
$$

subject to $A(0, \eta)=i \eta, B(0, \eta)=i \eta, C(0, \eta)=0$. 
It is easy to see that $A(\tau, \eta)=i \eta$. The solution for $B(\tau, \eta)$ is known analytically:

$$
B(\tau, \eta)=\frac{B_{1}-B_{2} G^{B_{1}-B_{2}} e^{\left(B_{1}-B_{2}\right) \tau}}{1-G^{B_{1}-B_{2}} e^{\left(B_{1}-B_{2}\right) \tau}}
$$

with $G=\left(\frac{i \eta-B_{1}}{i \eta-B_{2}}\right)^{\frac{1}{B_{1}-B_{2}}}$, where

$$
B_{1,2}=\frac{\alpha_{-}^{+} \sqrt{\alpha^{2}+2 \sigma^{2}\left(1-i \mu_{1} \eta\right)}}{\sigma^{2}}
$$

solves the quadratic equation $\frac{\sigma^{2} B^{2}}{2}-\alpha B+\mu_{1} A-1=0$.

For $C(\tau, \eta)$, we consider numerical solution since the Lévy exponents, $\psi(-i A)$ and $\psi(-i B)$ are complicated in nature for known Lévy processes. We use Runge-Kutta order 4 method, known to produce results that are close to analytical solutions.

\subsection{The Greeks}

In this subsection, we obtain closed-form formula for some of the sensitivities of the contingent claim, based on our model.

$\operatorname{delta}(\Delta):$

The delta of the option calculates how sensitive the option price is, with respect to the price of the asset. It is given as the partial derivative of the option price with respect to the asset price

$$
\begin{aligned}
\Delta & =\frac{\partial V(t, x, y)}{\partial S_{0}} \\
& =A \exp ((A-1) x+(B-1) y+C) .
\end{aligned}
$$

$\operatorname{gamma}(\Gamma)$ :

The gamma is the second order partial derivative of $V(t, x, y)$ with respect to the asset price

Therefore,

$$
\Gamma=\frac{\partial \Delta}{\partial S_{0}} .
$$

$$
\Gamma=A(A-1) \exp ((A-2) x+(B-2) y+C) .
$$

theta $(\Theta)$ :

The theta $\Theta$ of the option price is calculated as

$$
\begin{aligned}
\Theta & =\frac{\partial V(t, x, y)}{\partial T} \\
& =\left(A_{T} x+B_{T} y+C_{T}\right) \exp (A x+B y+C) .
\end{aligned}
$$



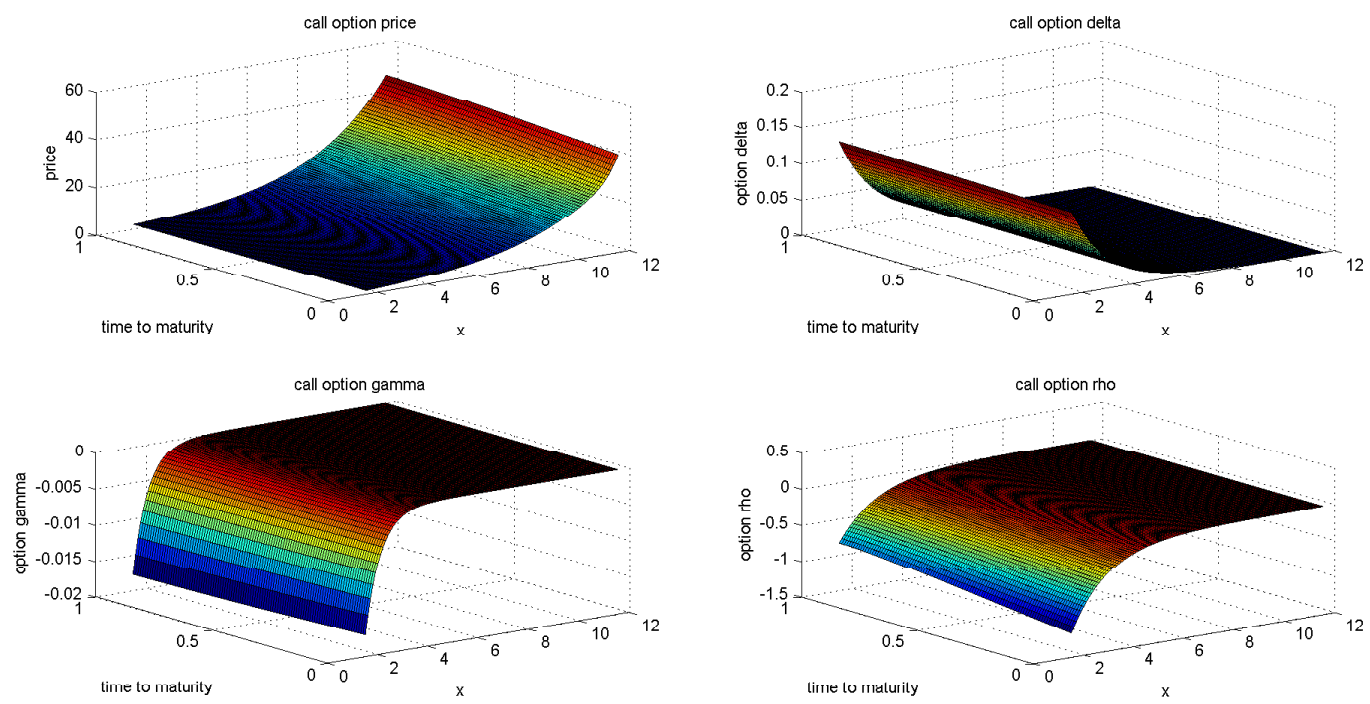

Figure 1: Call option values and its sensitivities

Rho $(\rho)$ :

The option $\operatorname{rho}(\rho)$ is the sensitivity of the option price with respect to the interest rate. Hence,

$$
\rho=\frac{\partial V(t, x, y)}{\partial r}
$$

and

$$
\rho=B \exp (A x+B y+C) .
$$

\section{Numerical Example and Conclusion}

In this section, we produce some numerical results based on the model proposed in this paper. We assume that the Lévy exponents $\psi_{x}(\eta)$ and $\psi_{y}(\eta)$ are given by $\psi(\eta)=c\left[\ln \left(-\lambda_{-}-i \eta\right)-\ln \left(-\lambda_{-}\right)+\ln \left(\lambda_{+}-i \eta\right)-\ln \left(\lambda_{+}\right)\right]$. The following parameters for the processes are used; $\lambda_{-}=-1, \lambda_{+}=1$, $c=0.025, \alpha=0.1, \beta=0.15, \sigma=0.5, \mu_{0}=15, \mu_{1}=0.25$. For this market, we calculate the price, delta, gamma and rho of European call option on the stock $S_{t}=e^{X_{t}+Y_{t}}$ with strike $K=100$ and maturity date $T=1$ year. The results of our calculations are presented graphically in Figure 1. 


\subsection{Concluding Remarks}

We have obtained analytical expressions for the European call option price and its sensitivities in Lévy models with jumps in interest rate. These expressions are easy to implement, and do not require numerical approximations.

\section{References}

[1] S. Asmussen, F. Avram, and M.R. Pistorious, Russian and American put options under exponential phase-type Lévy models, Stochastic Processes and its Applications 109 (2004), 79-111.

[2] O. Barndorff-Nielsen, Processes of normal inverse Gaussian type, Finance and Stochastics, 2 (1998), 41-68.

[3] F. Black and M. Scholes, The pricing of options and corporate liabilities, J. of Political Economy, 81 (1973), 637-654.

[4] M. Boyarchenko and S.Z. Levendorskii, Prices and sensitivities of barrier and first-touch digital options in Lévy-driven models, International J. of Theoretical and Applied Finance, 12, No 8 (2009), 1125-1170.

[5] S. Boyarchenko and S.Z. Levendorskii, American options in Lévy models with stochastic interest rate, Working Paper, http://papers.ssrn.com/abstract $=1015409$.

[6] S. Boyarchenko and S.Z. Levendorskii, American options in Lévy models with stochastic volatiity, Working Paper, http://papers.ssrn.com/abstract=1031280 (2008).

[7] J. Cox, J. Ingersoll Jr, and S. Ross, A theory of the term structure of interest rates, Econometrica, 53 (1985), 385-407.

[8] S.L. Heston, A closed-form solution for options with stochastic volatility with applications to bond and currency options. The Review of Financial Studies 6, No 2 (1993), 327-343.

[9] S.G. Kou, A jump diffusion model for option pricing, Management Science, 48 (2002), 1086-1101.

[10] S.Z. Levendorskii, Method of impaired contours and pricing of barrier options and CDS of long maturities, Working Paper, http://papers.ssrn.com/abstract=2267107 (2013). 
[11] D. Madan and F. Milne, Option pricing with V.G. martingale components, Mathematical Finance, 1, No 4, (1991), 3955.

[12] R.C. Merton, The theory of rational option pricing, Bell J. of Economics and Management Science 4 (1973), 1-19.

[13] R.C. Merton, Option pricing when the underlying stocks are discontinuous, J. of Financial Economics 5 (1976), 125-144.

[14] E.M. Stein and J.C. Stein, Stock price distribution with stochastic volatility: An analytic approach, The Review of Financial Studies 4, No 4 (1991), 727-752. 